\title{
ARTICLE
}

\section{Estimation of neutron duct streaming with the feeder for the power supply system in the JT-60 tokamak fusion device facility}

\author{
Atsuhiko M. Sukegawa ${ }^{a^{*}}$ and Koichi Okuno ${ }^{\mathrm{b}}$ \\ ${ }^{a}$ National Institute for Quantum and Radiological Science and Technology (QST), 801-1, Mukoyama, Naka-shi, Ibaraki-ken, \\ 311-0193, Japan; ${ }^{b} H A Z A M A-A N D O$ Corporation, 515-1, Karima, Tsukuba-shi, Ibaraki-ken, 305-0822, Japan
}

\begin{abstract}
The estimation of neutron duct streaming with a feeder for the power supply system in a JT-60 Tokamak fusion device facility has been performed via neutoronics analysis using a simplified formula for the cylindrical duct streaming model and a suitable model based on the Monte Carlo technique. However, the safety margin is too large for the evaluation using a simplified formula in order to evaluate the neutron streaming of a duct with three feeders (each feeder of size $0.126 \mathrm{~m} \times 0.357 \mathrm{~m} \times 2 \mathrm{~m}$ ) inside a cylindrical duct $(0.8 \mathrm{~m}$ diameter $\times 2 \mathrm{~m}$ length in size $)$. Further, a detailed evaluation of neutron streaming of a cylindrical duct with three feeders has not been performed in the facility. In this study, three-dimensional (3D) neutronics analysis using a real-model of the complex structured duct was performed to assess the safety margin of the simplified formula. It was confirmed that the neutron dose attenuation of the penetrating section in the duct using the simplified formula is approximately one order of magnitude larger than the real-model using the 3D calculation code.
\end{abstract}

Keywords: neutron duct streaming; Monte Carlo; Simon-Clifford formula; MCNP; PHITS; JT-60 Tokamak fusion device facility; concrete duct with metal feeder

\section{Introduction}

The upgrade of the JT-60 to the superconducting tokamak "JT-60SA" project is a combined EURATOM (EU)-Japan (JA) satellite tokamak under the Broader Approach agreement in support of the ITER project that supports research toward DEMO [1]. The assembly of the JT-60SA tokamak has started in January 2013.

In mainly deuterium-deuterium (DD) fusion discharges in JT-60 Tokamak fusion device, the reactions $\mathrm{d}(\mathrm{d}, \mathrm{n}) 3 \mathrm{He}$ and $\mathrm{d}(\mathrm{d}, \mathrm{p}) \mathrm{t}$ have equal probabilities, which indicates that tritons are generated at the same rate as the $2.45 \mathrm{MeV}$ neutrons in a DD plasma. The neutrons emitted by the DD fusion reaction in the plasmas are emitted from the vacuum vessel in a Tokamak fusion device.

The JT-60 Tokamak fusion device building is shielded by $2-\mathrm{m}$-thick ordinary concrete. Moreover, the wall and floor of the building have ducts for the piping and a cable of 40 places.

Figure 1 shows the typical design of a direct current (DC) feeder duct for toroidal field coils. Here we named "design A". In the design A, duct streaming calculation using the Simon-Clifford formula was carried out. The feeder was assumed to be air. The neutron shielding design of the duct is considered to be of twice-bending type using concrete. Therefore, the additional shield became large. The ratio of neutron dose $\left(\mathrm{H}_{\mathrm{n}} / \mathrm{H}_{0}\right)$ at the exit to that at the duct entrance is approximately $10^{-6}$. However, the neutron dose attenuation by the metallic feeder is not considered. Moreover, it is difficult to implement mockup tests using a neutron source in design $\mathrm{A}$.

In a previous neutron duct streaming experiment using ${ }^{252} \mathrm{Cf}$ neutron source, the experimental results were consistent with 3D Monte Carlo (3D MC) calculation using MCNP code [2]. The neutron spectrum of ${ }^{252} \mathrm{Cf}$ is a Maxwellian distribution with an average energy of approximately $2.1 \mathrm{MeV}$, and the neutron energy is relatively close to the energy of DD neutrons.

Therefore, we evaluate neutron duct streaming using 3D MC calculation to improve the design A. Further, the optimization of the additional shielding structure is required. In this paper, we discuss the following: the optimization of radiation shielding design of the duct by the estimation of neutron duct streaming considering metal in the duct using 3D MC calculation; a simplified formula for evaluating duct streaming with a metal feeder.

*Corresponding author. Email: morioka.atsuhiko@qst.go.jp 


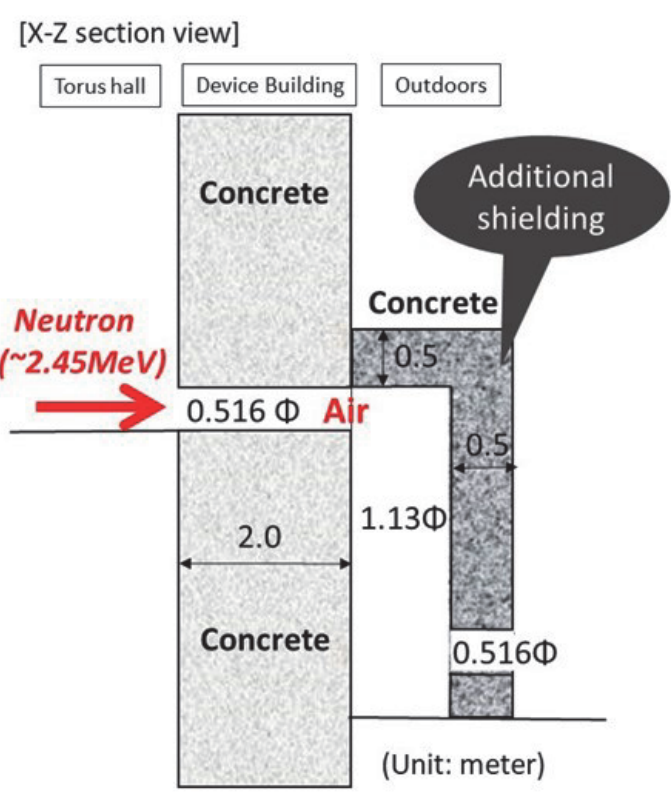

Figure 1. Design A of DC feeder duct.

\section{Duct with DC feeder of the facility}

Figure 2(a) shows the top view of a duct with a DC feeder for the power supply system in a JT-60 Tokamak fusion device facility. Figure 2(b) shows the cross-sectional view of the duct with the DC feeder. The diameter of the duct is $0.8 \mathrm{~m}$ and its length is $2 \mathrm{~m}$. There are three DC feeders for the toroidal magnetic field coil in the duct. The feeder has a cross section of $0.357 \mathrm{~m} \times$ $0.126 \mathrm{~m}$. The material of the feeder is copper. The gap between the three feeders and the duct is sealed with mortar.

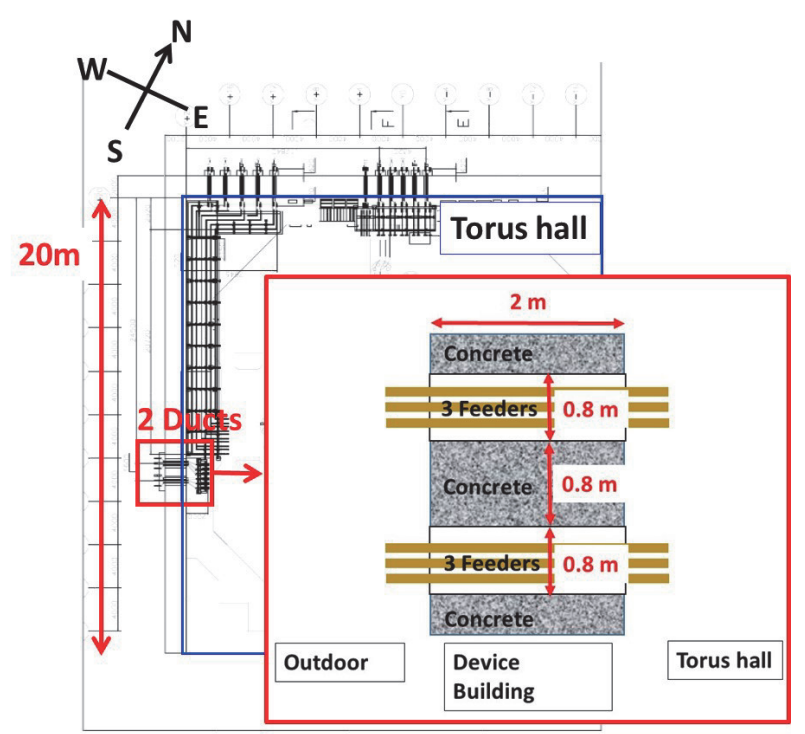

Figure 2(a). Top view of a typical duct with DC feeders for the power supply system in a Tokamak fusion device facility.

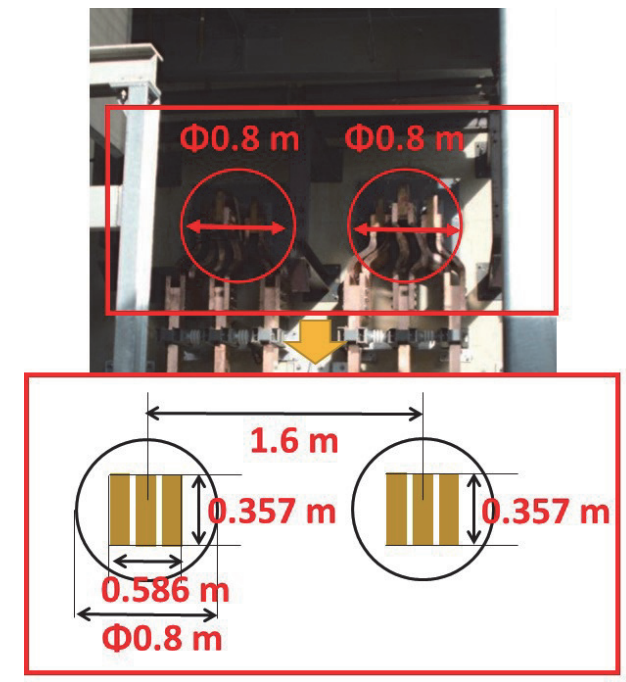

Figure 2(b). Cross-sectional view of duct with the DC feeder for the power supply system in JT-60 Tokamak fusion device facility.

\section{Estimation of the neutron duct streaming}

\subsubsection{Monte Carlo Calculation}

Neutron fluxes have been calculated using the PHITS code system [3], which couples the 3D MC code PHITS-2.88 (Ver.2.88) with the nuclear data library JENDL-4 [4]. In the model, the components are described by approximately 26 surfaces, which are combined into the specifications of approximately 50 cells. Figure 3 shows a cross-sectional view of the input geometry data for the PHITS analysis. The plane source is set at $\mathrm{z}=0$ as the neutron source of mono-energetic $2.45 \mathrm{MeV}$ neutron.
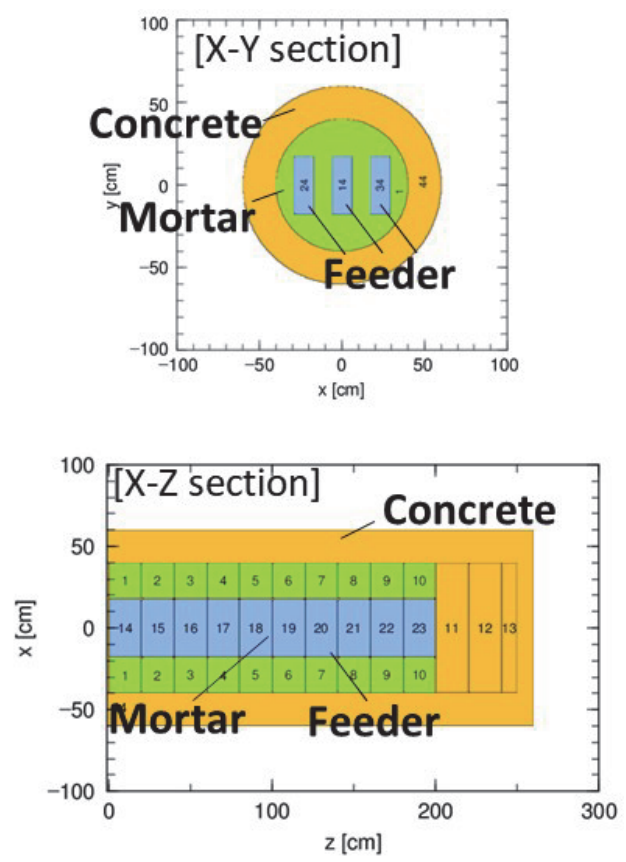

Figure 3. Cross-sectional view of the 3D model for neutron duct streaming with the three metallic feeders. 
The neutron dose has been calculated in the duct with a feeder using a surface crossing estimator ([t-cross] tally) and track length estimator ([t-track] tally). Neutron fluency energy spectra (100 energy-groups from $10^{-8}$ $\mathrm{MeV}$ to $20 \mathrm{MeV}$ ) have been calculated using PHITS. In this calculation, histories are $10^{8}$ neutrons. We made use of variance reduction using the weight window technique. Figure 4(a) shows a neutron dose contour map using PHITS analysis. Moreover, Figure 4(b) shows a relative error contour map using PHITS analysis.

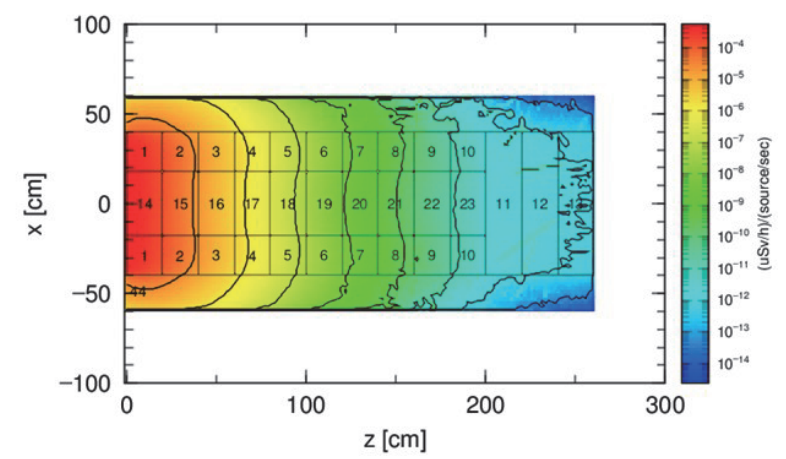

Figure 4(a). Neutron dose contour map.

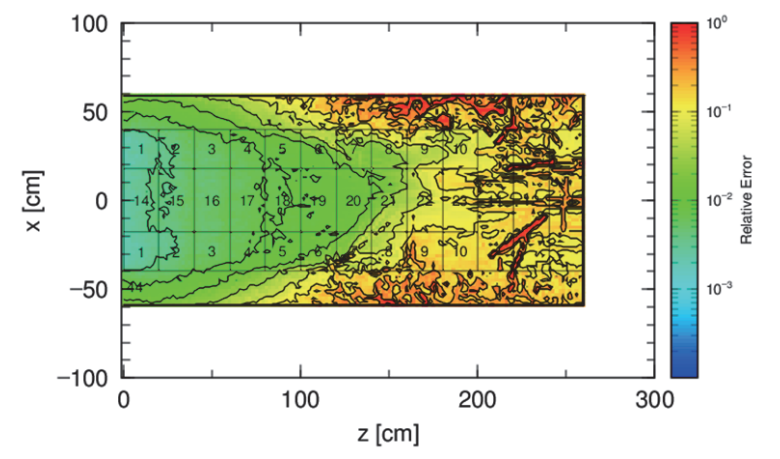

Figure 4(b). Relative Error contour map.

\subsection{Simplified formula for duct streaming}

\subsubsection{Simon-Clifford formula}

In the design A, duct streaming calculation using the Simon-Clifford formula [5] is carried out and the feeder is assumed to be air. Figure $\mathbf{5}$ shows the cross-sectional view of the twice-bending duct. The formula is expressed by Eq. (1).

$$
\frac{H_{n}}{H_{0}}=C\left(\frac{d_{0}^{2}}{8 l_{0}^{2}}\right)\left(\frac{\alpha d_{1}^{2}}{8 l_{1}^{2} \sin \theta_{1}}\right) \cdots \cdots . .\left(\frac{\alpha d_{n}^{2}}{8 l_{n}^{2} \sin \theta_{n}}\right)
$$

where $H_{n}$ is the dose at the exit point, $H_{0}$ is the dose at the incidence point, $d_{j}\left(=\operatorname{root}\left(4 a_{j} b_{j} / \pi\right)=\operatorname{root}(4 \times 0.586\right.$ $\mathrm{m} \times 0.357 \mathrm{~m} / 3.1415)=0.516 \mathrm{~m})$ is the equivalent diameter, $l_{i}$ is the duct length, $\theta$ is the angle of the bend (=90), $\alpha$ is the albedo ( $=0.7$ for concrete) [6], $C$ is the safety factor $(=4)$, and $a_{j}$ and $b_{j}$ are the dimensions of the duct. Figure 6 shows the concept of equivalent diameter of three feeders in the duct. By considering the mortar of the feeder and the feeder itself as a metallic feeders, the estimation of neutron streaming has a safety margin.

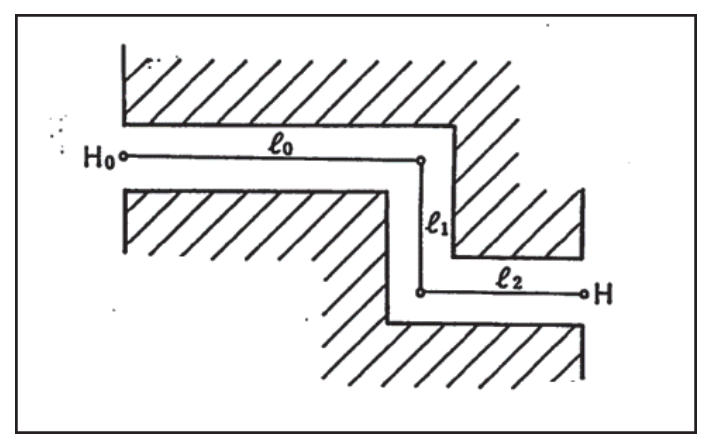

Figure 5. Cross-sectional view of the twice-bending duct.

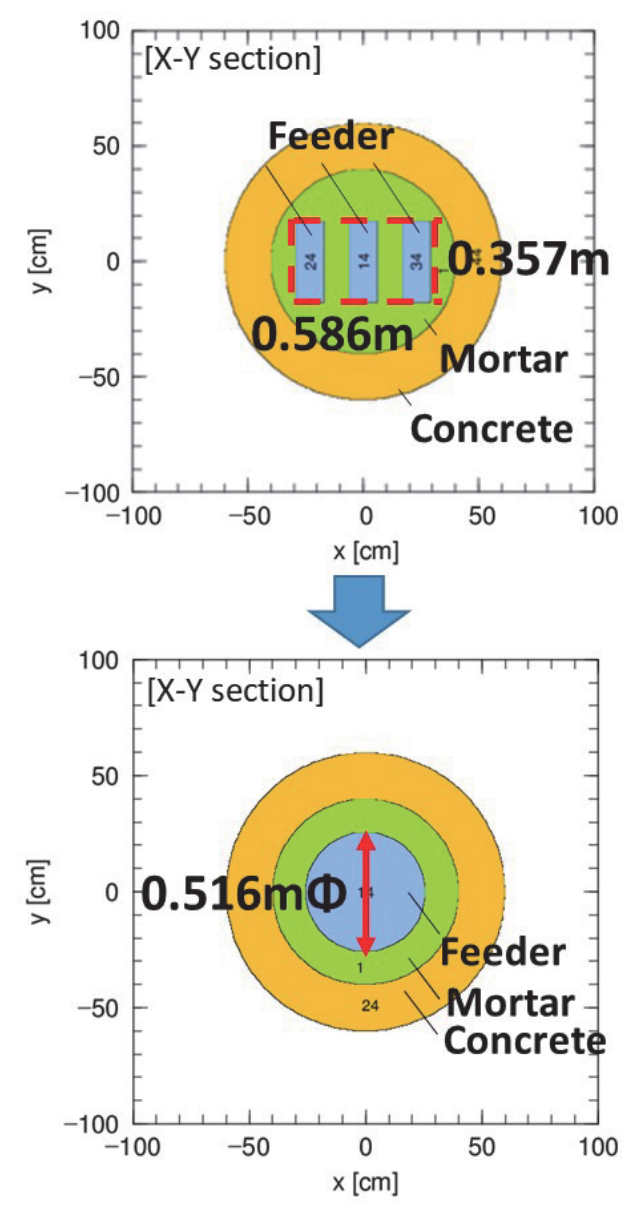

Figure 6. Concept of the equivalent diameter of three feeders.

\section{Results and discussion}

Figure 7 shows the neutron dose attenuation by neutron streaming effects with three feeders in the duct. The black square symbols indicate the result of the duct streaming with the feeder using the 3D MC method. The white square symbols indicate the result of the duct streaming with the feeder of the equivalent diameter using the 3D MC method. The circular symbols indicate the result of the duct streaming without the feeder using 
the 3D MC method. Furthermore, the triangular symbols indicate the results of the Simon-Clifford formula in the design A. In these estimations of duct streaming with/without feeder using the 3D MC method, the design value $\left(\mathrm{H}_{\mathrm{n}} / \mathrm{H}_{0}\right.$ of approximately $\left.10^{-6}\right)$ of the neutron dose attenuation of the duct with feeder is cleared with a path length of $2 \mathrm{~m}$. However, the design A of duct using the Simon-Clifford formula requires a path length of approximately $6.6 \mathrm{~m}$.

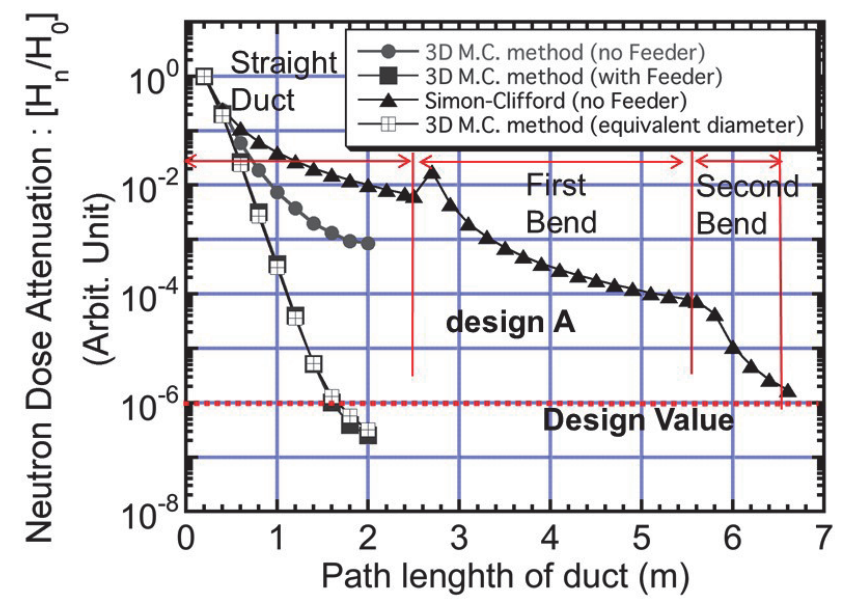

Figure 7. Neutron dose attenuation of with three feeders in the duct.

In this study, it is observed that neutron dose attenuation by the duct with a feeder decreases by approximately 6 orders of magnitude compared to the use of no feeders. Therefore, the neutron shielding design of the duct with a metal feeder has been improved. Figure 8 shows the improved design for the DC feeder duct without an additional shielding on the design A.

\section{Conclusion}

The estimation of neutron duct streaming with a feeder for the power supply system in a JT-60 Tokamak fusion device facility was performed via neutronics analysis using the 3D Monte Carlo technique.

A 3D neutronics analysis using a model for the duct with metal feeders was carried out. It was verified that the neutron dose attenuation in the duct using the simplified formula was approximately one order of magnitude larger than that of the real-model using the $3 \mathrm{D}$ calculation code.

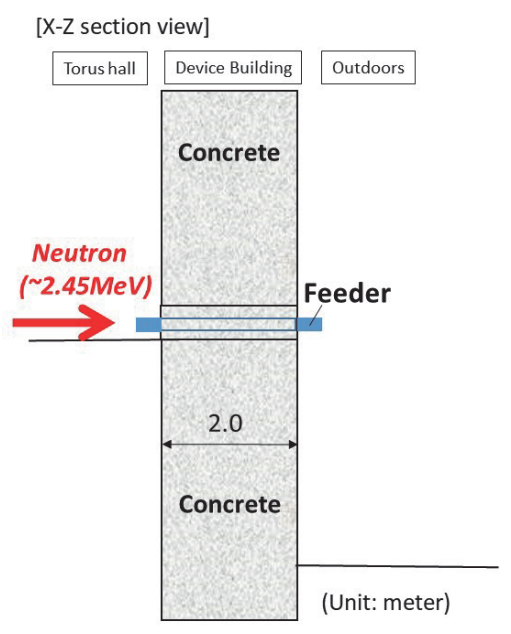

Figure 8. Improved design of the DC feeder duct.

\section{Acknowledgements}

We would like to acknowledge Dr. M. Matsukawa for his kind suggestions for the maintenance of this power supply system. We would like to acknowledge Dr. Y. Hirao and Dr. N. Matsuda for some suggestions for the simplified formula of duct streaming. The authors would like to thank PHITS development team for their contribution to this streaming analysis using 3D Monte Carlo calculation technique.

\section{References}

[1] S. Ishida, et al, Status and prospect of the JT-60SA project, Fusion Engineering and Design. (2010) pp.2079-2804.

[2] T. Miura, Y. Hirao, N. Odano, et al., Experimental Study on the Assessment of Safety Allowance Taken in Shield Irregularity Design Method, NMRI Technical Report, 2 (1) (2002). [in Japanese]

[3] T. Sato, K. Niita, N. Matsuda, et al., Particle and Heavy Ion Transport Code System PHITS, Version 2.52, J. Nucl. Sci. Technol. 50:9 (2013), pp. 913-923.

[4] K. Shibata, O. Iwamoto, T. Nakagawa, et al., JENDL-4.0: A New Library for Nuclear Science and Engineering, J. Nucl. Sci. Technol. 48:1 (2011), pp. 1-30.

[5] A. Simon and C.E. Clifford, The attenuation of neutrons by air ducts in shields, Nuclear Science and Engineering., 1 (1956), pp.156-166.

[6] W.E. Selph, NEUTRON AND GAMMA-RAY ALBEDOS, OAK RIDGE NATIONAL LABORORATORY, ORNL-RSIC-21 (1968). 